\title{
Optoelectronic and nonlinear optical processes in low dimensional semiconductors
}

\author{
B P SINGH \\ Department of Physics, Indian Institute of Technology Bombay, Mumbai 400 076, India
}

\begin{abstract}
Spatial confinement of quantum excitations on their characteristic wavelength scale in low dimensional materials offers unique possibilities to engineer the electronic structure and thereby control their physical properties by way of simple manipulation of geometrical parameters. This has led to an overwhelming interest in quasi-zero dimensional semiconductors or quantum dots as tunable materials for multitude of exciting applications in optoelectronic and nonlinear optical devices and quantum information processing. Large nonlinear optical response and high luminescence quantum yield expected in these systems is a consequence of huge enhancement of transition probabilities ensuing from quantum confinement. High quantum efficiency of photoluminescence, however, is not usually realized in the case of bare semiconductor nanoparticles owing to the presence of surface states. In this talk, I will focus on the role of quantum confinement and surface states in ascertaining nonlinear optical and optoelectronic properties of II-VI semiconductor quantum dots and their nanocomposites. I will also discuss the influence of nonlinear optical processes on their optoelectronic characteristics.
\end{abstract}

Keywords. Optoelectronic process; nonlinear optical process; semiconductor.

Quest for ever faster and intelligent information processing technologies has sparked great scientific interest in information as a physical entity and its related hardware. Since any information can be represented as a twodimensional pattern in some physical domain or other, pattern generation and recognition then becomes the central theme in information processing. Its basic functions viz. generation, transaction, transportation, transformation, compression and storage, can all be realized by nonlinear dynamical systems as these exhibit self organization through spatio-temporal instabilities leading to spontaneous pattern generation. Ability of photons to carry information in massively dense parallel streams and to interact in nonlinear medium makes nonlinear optical systems an ideal choice for information processing hardware. Simplest example is the system consisting of a thin nonlinear optical medium (Kerr-slice) and a feedback mirror. Instrumentation requirement of such machines will involve optoelectronic and nonlinear optical devices. No technology succeeds and matures without the availability of task specific materials. Unfortunately, till date we do not have materials with proper mix of linear and nonlinear optical properties despite investigations on enormous variety of materials. It then becomes imperative to develop device grade nonlinear and optoelectronic materials via electronic structure engineering. Two classes of materials are lucrative for this purpose, viz. $\pi$-conjugated organic polymers and inorganic semiconductors due to large electron

(bhanups@phy.iitb.ac.in) delocalization favouring large optical nonlinearities in these systems. While the former one possesses enormous scope for property engineering by alterations in their chemical structure, semiconductors though have well developed technology for the fabrication and integration of devices do not appear to have the same advantage. As a consequence of electron motion in periodic potential of semiconductor crystals, their electronic structure comprises of conduction and valence bands separated by forbidden energy gap. Electron, hole and their composite bound state called exciton constitutes the relevant elementary excitations in semiconductors. Spatial extension of excitons characterized by exciton-Bohr radius depends upon the extent of coulomb screening in a given semiconductor. Typical size of excitons in some of the semiconductors is shown in table 1 .

If these quantum entities were to be confined by a potential on the length scale of exciton-Bohr radius ( $\sim$ few $\mathrm{nm})$ but larger than the lattice constant, one would expect marked influence of the spatial nature-size, shape and barrier height — of the confining potential on their motion and hence the optical and electronic properties of semiconducting materials. One way to realize this is to synthesize semiconductor nanocrystallites. As the motion of electron and hole is quantum confined in all three directions, semiconductor nanoparticles are also called quantum dots. For the sake of simplicity we consider a spherical nanocrystallite. Two-particle Hamiltonian for such a system with kinetic energy terms, coulomb potential and confinement potential, $U(r)$, may be written as (Woggon 1997) 
Table 1. Exciton size for various semiconductors.

\begin{tabular}{lc}
\hline Semiconductor & Exciton Bohr radius $(\mathrm{nm})$ \\
\hline $\mathrm{ZnO}$ & 2 \\
$\mathrm{CdS}$ & 3 \\
$\mathrm{GaAs}$ & 12 \\
$\mathrm{InAs}$ & 38 \\
\hline
\end{tabular}

$$
H=-\left(\hbar^{2} / 2 m_{\mathrm{e}}\right) \nabla_{\mathrm{e}}^{2}-\left(\hbar^{2} / 2 m_{\mathrm{h}}\right) \nabla_{\mathrm{h}}^{2}-\frac{e^{2}}{2 \varepsilon\left|r_{\mathrm{e}}-r_{\mathrm{h}}\right|}+U(r),
$$

where $m_{i}, r_{i}$ for $i=\mathrm{e}$ and $\mathrm{h}$ refer to the mass and coordinates of the electron and hole, respectively. In case, the dot size, $a$, is larger than the exciton-Bohr radius, $a_{\mathrm{B}}\left(a \geq a_{\mathrm{B}}\right)$, referred to as weak confinement limit, quantization of the centre of mass motion occurs. Energy of the lowest state can be expressed as

$$
E_{1 s 1 s}=E_{\mathrm{g}}-R_{\mathrm{y}}^{*}\left\{1-\frac{\mu}{M}\left(\frac{\pi a_{\mathrm{B}}}{a}\right)^{2}\right\},
$$

where $R_{\mathrm{y}}^{*}$ is the exciton Rydberg energy, $\mu$ the electronhole reduced mass and $M=m_{\mathrm{e}}+m_{\mathrm{h}}$. Here, the first exciton resonance experiences blue shift in the energy that is small compared to the binding energy, $R_{\mathrm{y}}^{*}$.

On the other hand, when the particle radius is small compared to exciton Bohr radius, $\left(R \leq a_{\mathrm{B}}\right)$, electron-hole pair is said to be strongly confined. In this case, even though the electron and hole are confined within a nanoparticle, these do not form a bound state because of large zero point energy. Presence of $U(r)$ term in the Hamiltonian does not allow the centre of mass motion and the motion of a particle with reduced mass is to be considered independently. The ground state $(1 s 1 s)$ energy of electronhole pair, in this case, can be expressed as

$$
E_{1 s 1 s}=E_{\mathrm{g}}+\frac{\hbar^{2} \pi^{2}}{2 \mu a^{2}}-\frac{1 \cdot 786 e^{2}}{\varepsilon a}-0 \cdot 248 R_{\mathrm{y}}^{*} .
$$

In the strong confinement regime, blue shift in band gap energy is quite significant compared to weak confinement limit. Along with the blue shift of the band gap and excitonic levels, particle size reduction also causes an enhancement in the oscillator strengths $\left(f_{\mathrm{qd}}\right)$. This can be understood as follows. Optical transition probability in a bulk semiconductor is proportional to the probability of finding an electron and a hole in the same unit cell of the crystal. Hence, the oscillator strength of the exciton, $f_{\mathrm{exc}}$, is inversely proportional to $a_{\mathrm{B}}^{3}$. In the case of quantum dots transition probability is proportional to the spatial restriction of carrier motion in the quantum dot volume due to externally imposed quantum confinement. Comparing the two, we get

$$
\frac{f_{\mathrm{qd}}}{f_{\mathrm{exc}}}=a_{\mathrm{B}}^{3} / a^{3} .
$$

Equation (4) suggests large enhancement in the oscillator strength of quantum dots in the strong confinement limit.

It thus appears from the above discussion that one can engineer the electronic structure and properties of semiconductors simply by manipulating the geometrical parameters such as size and shape of the nanocrystallites. Large oscillator strengths of quantum dots realizable in the strong confinement limit will manifest in the enhanced radiative decay rates leading to high photoluminescence yield and in the enhanced nonlinear optical susceptibilities and become lucrative for their application in optoelectronic and nonlinear optical devices. However, due to the dangling bonds at surface of nanocrystallite, the advantage of high oscillator strength is offset by the presence of surface electronic states within the HOMO-LUMO gap that provide competing non-radiative decay channels and lead to severe degradation in optoelectronic properties. This problem has been addressed by capping the semiconductor nanoparticles with a thin layer of higher band gap material (Woggon 1997). Many techniques based on wet chemical synthesis using organic functional groups can produce capped nanoparticles. However, these are not very useful for fabricating large area thin films that can be integrated into devices. Surface states can also be passivated by embedding nanoparticles in higher band gap host matrix. The interface between the nanoparticle and matrix introduces impurity states that alter carrier dynamics of the quantum dot and affect its optical response in nontrivial manner. Other rather complicated multi-step techniques have been developed to produce core shell structures. Therefore, a simple, single step, solid state technique that produces a uniform, surface passivated nanocrystalline thin film with good adhesion to the desired substrate will be of great value.

Nanocrystalline films of most metals, semiconductors and oxides can be sputtered onto desired substrates by d.c./rf sputtering. Nanostructure formation is promoted by carrying out the sputtering at relatively high gas pressures (20-200 mTorr) and low substrate temperatures (100$300 \mathrm{~K})$. We have grown the $\mathrm{CdS}$ and $\mathrm{ZnO}$ nanoparticle thin films rf-sputtering typically in 100-200 mTorr Ar, on quartz and Si substrates maintained at $300 \mathrm{~K}$ (Ayyub et al 2001). The particle size distribution obtained from absorption X-ray diffraction line shape analysis was $4 \cdot 8 \pm 2 \mathrm{~nm}$. Surface states probably can be passivated by forming a nanocomposite having a random distribution of two different band gap semiconductors at nanometer scale. We, therefore, attempted to passivate surface states through the formation of nanocomposite films by co-sputtering $\operatorname{CdS}\left(E_{\mathrm{g}}=2.5 \mathrm{eV}\right)$ together with $\mathrm{ZnO}\left(E_{\mathrm{g}}=3.3 \mathrm{eV}\right)$ from a single, composite target (Taneja et al 2002). We used targets with $\mathrm{CdS}: \mathrm{ZnO}$ mole fractions of 0.45 ( $n$-CZ1), 0.40 (n-CZ2) and 0.33 (n-CZ3). Transmission electron micrograph (TEM) of these films in figure 1 show a nearly uniform distribution of closely aggregated, individual $\mathrm{CdS}$ and $\mathrm{ZnO}$ nanoparticles with an average size of about 
$3 \mathrm{~nm}$ for both components. Our studies also show that the particle size distribution can be significantly reduced by co-sputtering two compounds. The size distribution in the $\mathrm{CdS}: \mathrm{ZnO}$ nanocomposite was only $\pm 1 \mathrm{~nm}$ (about $3 \mathrm{~nm}$ ). Figure 2 shows the absorption spectra of $\mathrm{CdS}$ and $\mathrm{CdS}-$ $\mathrm{ZnO}$ nanocomposite films (Vasa et al 2002). Blue shift of the band gap as indicated by the absorption edge with the reduction in the particle size for pure $\mathrm{CdS}$ and nanocomposite $\mathrm{CdS}-\mathrm{ZnO}$ thin films is clearly evident. Thus the band gap can be tuned merely by controlling the size of the nano particles.

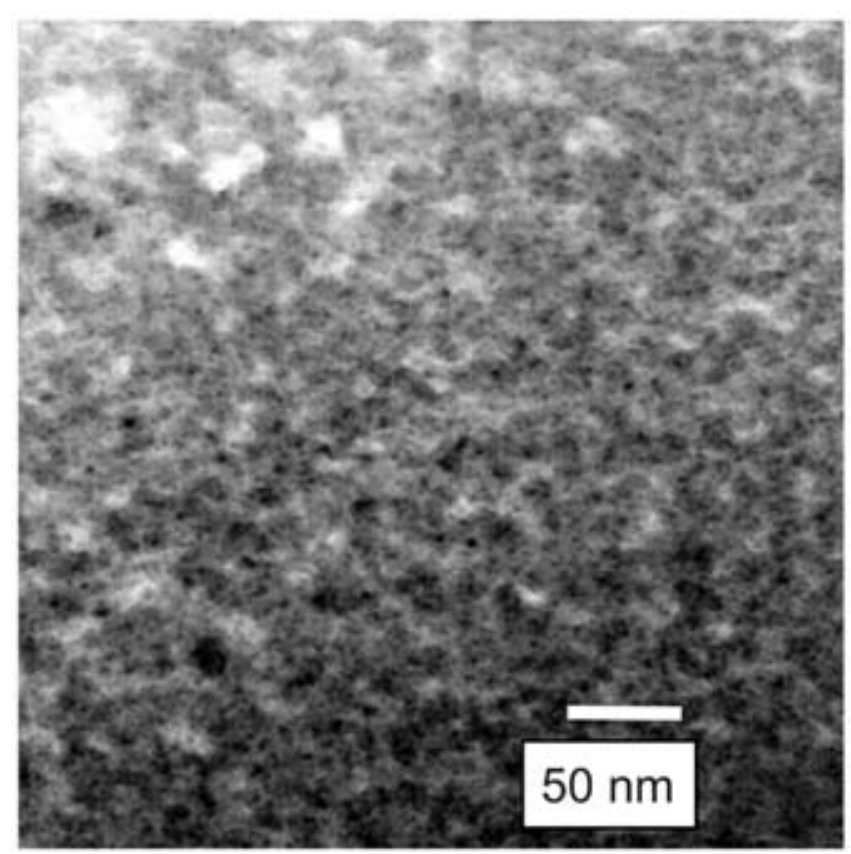

Figure 1. A transmission electron micrograph of a typical $\mathrm{CdS}-\mathrm{ZnO}$ nanocomposite thin film deposited on $\mathrm{Si}$.

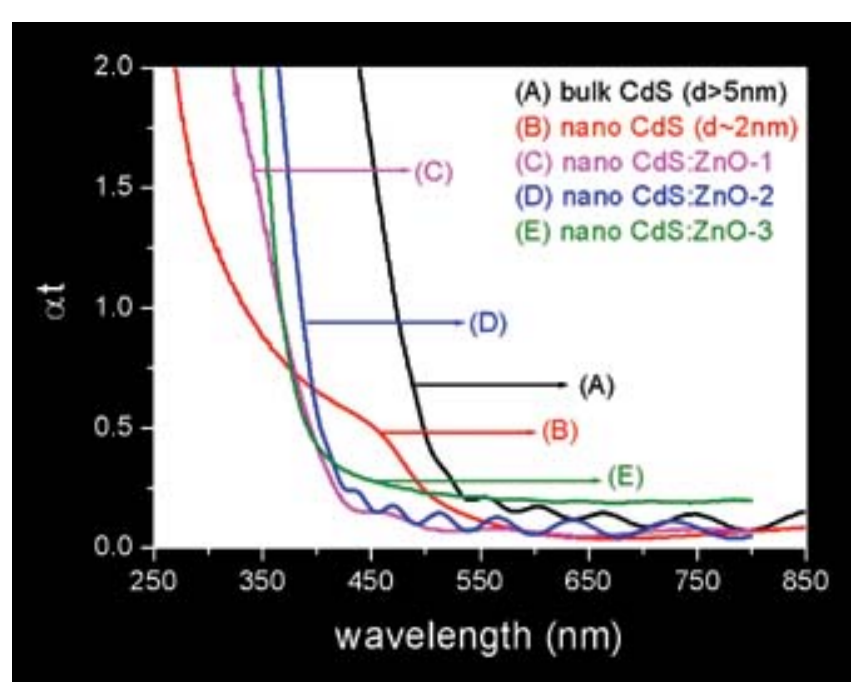

Figure 2. Absorption spectra of pure nano $\mathrm{CdS}$ and $\mathrm{CdS}-\mathrm{ZnO}$ nanocomposite thin films.
Photoluminescence (PL) spectra of these samples (Ayyub et al 2005) is shown in figure 3. Due to the probable presence of a large number of surface states, we observed a very weak, broad-band PL from these films at room temperature when excited by the second harmonic of a Ti:sapphire laser system with the fundamental of $800 \mathrm{~nm}$. No specific transitions were observed and the spectrum was red shifted, indicating the involvement of non-radiative transitions. The general characteristics of the emitted PL from the nanocomposite films were similar to those from bulk CdS films, indicating that the same emission mechanism was involved in each case. Significantly, we observed an enhancement in the PL yield by atleast two orders of magnitude from the $n$-CZ2 thin film compared to bulk CdS thin films. The PL enhancement for the same sample compared to nano CdS films was about six-fold. These observations, therefore, demonstrate that by co-sputtering two semiconductors in a suitable ratio, it is possible to effectively passivate semiconductor nanoparticles in a single step process.

The resonant one-photon nonlinearity of the nanocrystalline samples was studied using an excitation wavelength of $\sim 390 \mathrm{~nm}$, which is close to the absorption edge in most of our samples (Vasa et al 2005). The intensity of the beam on the sample from the second harmonic of a Ti : sapphire laser ( $80 \mathrm{fs}, 100 \mathrm{MHz}, \sim 20 \mathrm{~mW}$ ) could be changed by several orders of magnitude by translating a focusing lens along the $z$-axis ( $z$-scan). From a study of the intensity dependence of PL, we observed a completely reversible quenching of PL (figure 4). When the sample was at the focal point (highest average intensity, $\sim 10 \mathrm{KW} / \mathrm{cm}^{2}$ ), the PL output got reduced by $\sim 80 \%$. A comparison of PL spectra recorded at and away from the focal point indicates that the spectral features remained unchanged (figure 4 inset), except for a change in the intensity. A similar result was obtained when the intensity was changed by varying the incident power instead of the spot size.

A quenching of the PL may arise from a number of different origins. It is possible that the damage threshold is crossed at higher intensities leading to a reduction in the PL yield. There are many reports of photodarkening in which the sample shows either an irreversible damage or a very slow recovery. However, since the effect is completely reversible in our samples, the intensity is certainly below the damage threshold. The other possibilities include: (i) saturation of absorption at pump wavelengths, (ii) re-absorption of the PL wavelengths either by free carrier absorption (FCA) or (iii) re-absorption by ESA. To probe this, we again performed z-scan at $390 \mathrm{~nm}$, but recorded the transmission instead of the PL. The transmission was also seen to get reduced at the focal point. This rules out the possibility of saturation of absorption as, in that case, the transmission should have increased at the focal point. The two remaining possibilities for quenching of PL are FCA and ESA. 


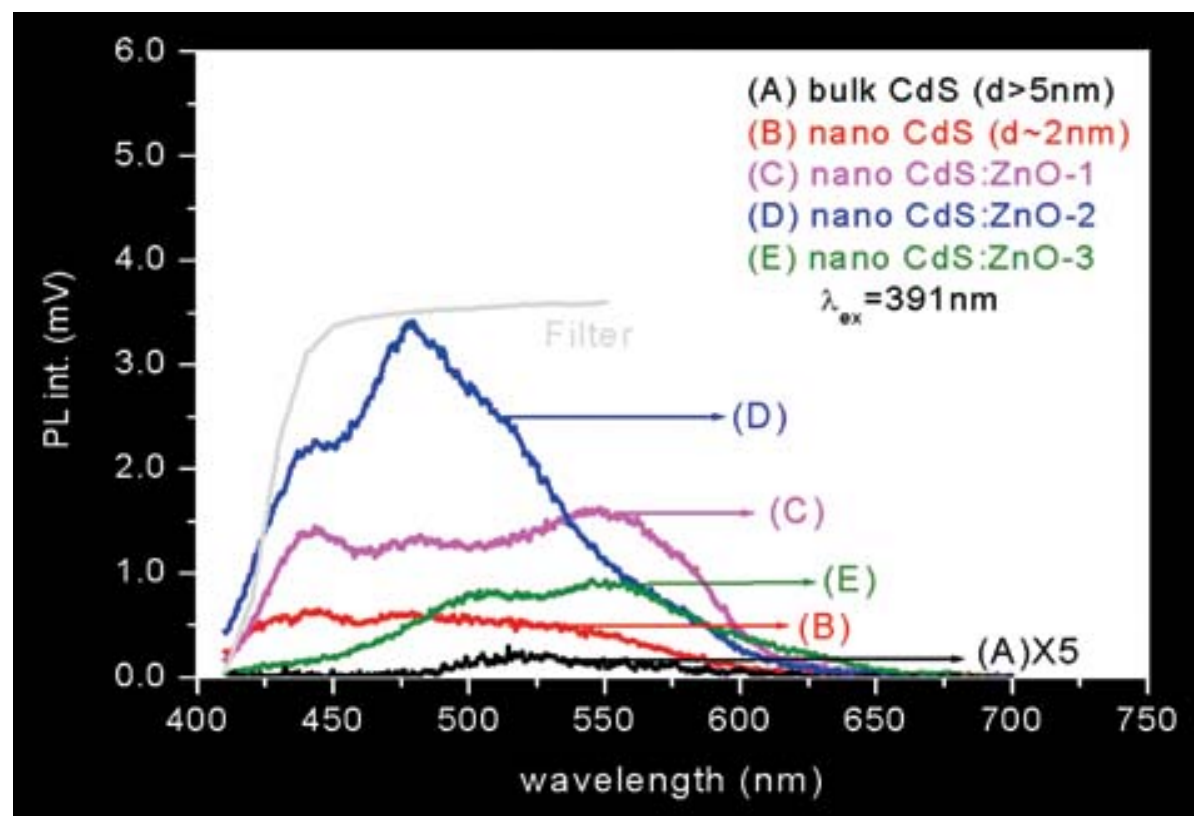

Figure 3. Photoluminescence spectra of pure nano $\mathrm{CdS}$ and $\mathrm{CdS}-\mathrm{ZnO}$ nanocomposite thin films.

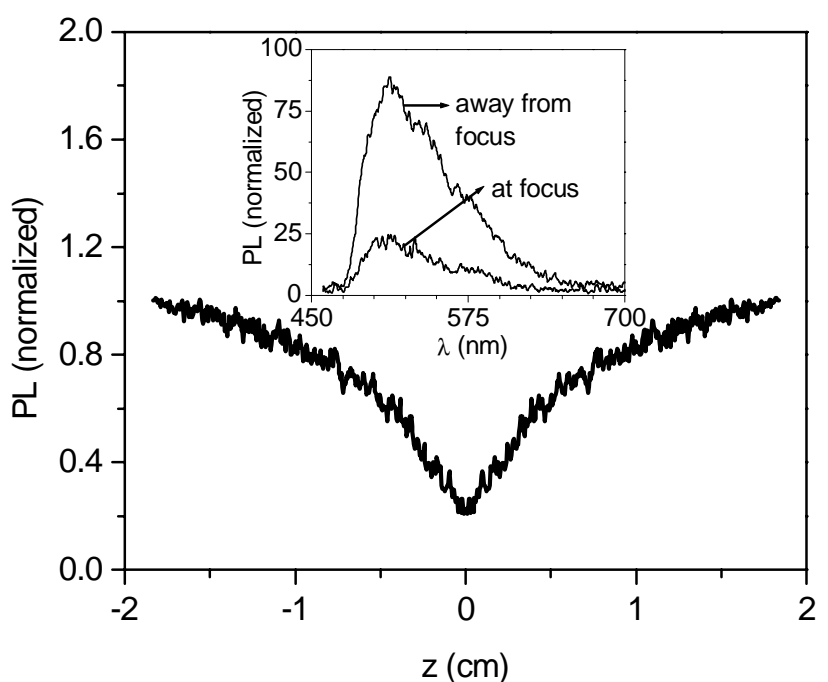

Figure 4. Photoluminescence emission from a nano $\mathrm{CdS}: \mathrm{ZnO}(n-\mathrm{CZ2})$ thin film at $591 \mathrm{~nm}$ as a function of incident intensity, excited by the second harmonic of a $\mathrm{Ti}$ : sapphire laser $(\sim 390 \mathrm{~nm}, 80 \mathrm{fs}, 100 \mathrm{MHz}, 40 \mathrm{~mW})$ and collected normal to the incident direction. Inset shows the PL spectra for the same sample, recorded from points at and away from the focus (which are, therefore, excited at different intensities).

The nonlinear absorption coefficient, $\beta_{\text {eff }}$, was calculated for the bulk CdS, nano-CdS and nanocomposite films from the intensity-dependence of the transmission. The nanocomposite with the highest PL also showed the highest $\beta_{\text {eff }}(16,500 \mathrm{~cm} / \mathrm{GW})$, indicating that nanocomposite formation not only affects PL properties but also important nonlinear properties. Photodarkening was also observed in the nano and bulk CdS films, implying that the relevant electronic states are associated with $\mathrm{CdS}$ and not with the matrix interface, as in semiconductor-doped glasses. Due to the very high linear absorption, it was not possible to measure $\beta_{\text {eff }}$ in bulk CdS films. ZnO does not absorb appreciably at the excitation wavelength and shows no nonlinearity at the operating power.

Non-degenerate pump-probe spectroscopy was used to study the carrier dynamics and determine the origin of intensity dependent absorption (Vasa et al 2005). A schematic of the experimental set up is shown in figure 5 .

The pump beam was obtained from the second harmonic of a Ti : sapphire laser and was modulated at $121 \mathrm{~Hz}$. A multi-line $\mathrm{Ar}^{+}$laser $(\sim 12 \mathrm{~mW})$ was used to generate the probe beam. In the presence of the pump beam, the transmission of the probe beam decayed (due to generation of charge carriers) with a time constant, $\sim 2 \mathrm{~ms}$ (figure 6 , squares, top). In its absence, the transmission grew (due to relaxation of charge carriers) with a similar time constant (figure 6, squares, bottom). The sample with the highest PL showed faster decay and growth.

Earlier, we had also observed a significant photocurrent in these films at UV-visible wavelengths in presence of a bias voltage $>100 \mathrm{~V}$ (Vasa et al 2002). On repeating the pump-probe experiment in the presence and absence of a bias voltage $(282 \mathrm{~V})$, no change was observed in the generation and relaxation time of the excited carriers. This result confirmed that the nonlinear absorption in our samples is caused by ESA and not FCA. Since the time scale relevant to our samples is $\sim 1 \mathrm{~ms}$, the $100 \mathrm{MHz}$ wave train of 80 fs pulse duration (average power, $\sim 10 \mathrm{~mW}$ ) is essentially equivalent to a $\mathrm{CW}$ beam with an average 
power of $10 \mathrm{~mW}$. We also perfomed $z$-scan with a solid state $\mathrm{CW}$ diode laser $(402 \mathrm{~nm}, \sim 10 \mathrm{~mW})$. Similar results were obtained, indicating that the nonlinear absorption is fluence dependent and not intensity dependent.

In the nanocomposite films, the relevant states are intrinsic to the CdS QDs and their interactions are modified by the presence of $\mathrm{ZnO}$. The linear and nonlinear properties of nanocrystalline $\mathrm{CdS}$ and nanocomposite $\mathrm{CdS}: \mathrm{ZnO}$ films can be satisfactorily explained in terms of the fourlevel model described below.

Let us assume that there are two pairs of levels that cannot be coupled to each other by radiative transitions due to the selection rules. Levels other than the HOMO and LUMO bands are generated due to either $\mathrm{Cd}$ or $\mathrm{S}$ vacancy or by other defect states. All the levels are consi-

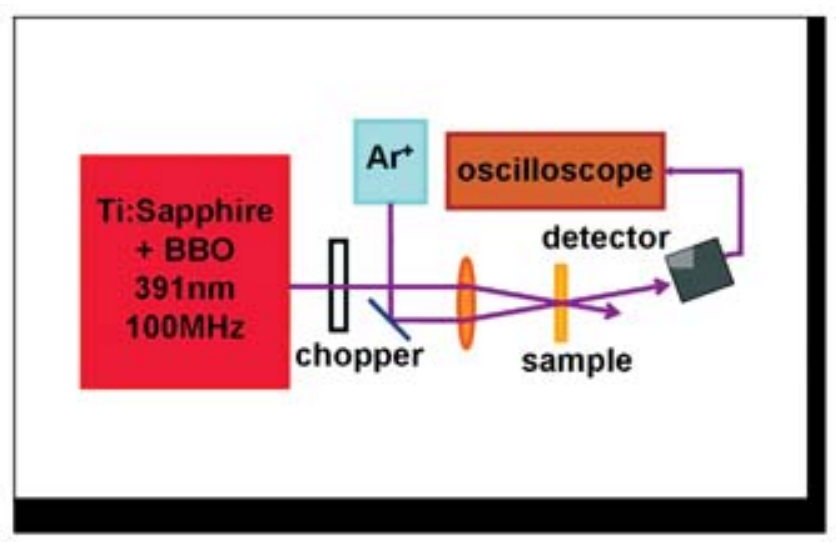

Figure 5. Experimental set up for pump probe spectroscopy.

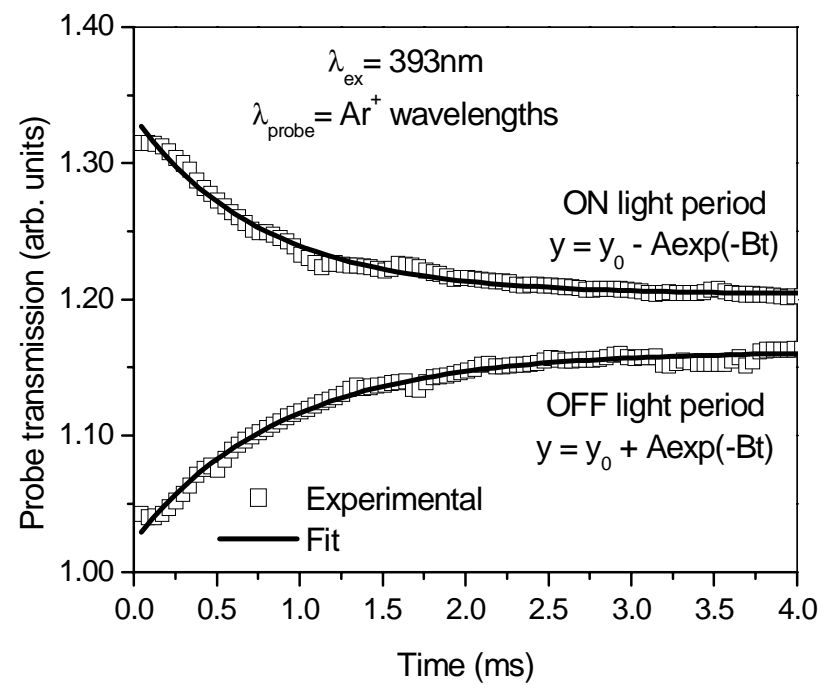

Figure 6. Transmission of the probe beam (multiline $\mathrm{Ar}^{+}$laser, $12 \mathrm{~mW}$ ) in the presence and absence of the pump beam (second harmonic of Ti : sapphire: $390 \mathrm{~nm}, 80 \mathrm{fs}, 100 \mathrm{MHz}, 20 \mathrm{~mW}$ ) for nano $\mathrm{CdS}: \mathrm{ZnO}(n-\mathrm{CZ2})$. The accuracy in the response time measurement is $\pm 10 \%$. Solid line shows the fit based on a semiquantitative 4-level model. derably broadened because of a distribution in the particle size. One of the pairs consist of HOMO band acting as a ground state $\left(N_{1}\right)$ and LUMO band states lying close to it acting as excited state $\left(N_{3}\right)$. The other pair of levels consists of states generated by impurity or defect states $\left(N_{2}\right.$ and $N_{4}$ ). The positions of these energy levels shown in the schematic diagram (figure 7) are notional and do not denote the actual energy scale.

When the sample is excited by a wavelength close to the band gap, the population is transferred from the HOMO to the LUMO band. The excited carriers relax rapidly to closely lying states by a fast nonradiative transition occurring over $\sim$ ps time scale. From these states, they either relax to HOMO levels by a radiative transition with a decay rate, $\gamma_{\mathrm{a}}$ or are transferred to $N_{2}$ by a nonradiative transition (with decay rate, $\gamma_{b}$ ) occurring over $\sim 10$ ps time scale. The relatively weak and red-shifted radiative transition from $N_{3}$ to $N_{1}$ with a quantum efficiency of about $0.1 \%$ corresponds to the observed PL transition and the effective relaxation time varies from 50-300 ps depending on the sample. $n$-CZ2 has the largest PL quantum yield and shows the fastest effective relaxation time of less than $50 \mathrm{ps}$ (table 1). Carriers relax from $N_{2}$ to $N_{1}$ by a relatively slow non-radiative transition (with decay rate, $\gamma_{c}$ ) at a $\sim$ ms timescale, leading to a higher density of charge carriers in $N_{2}$. As the population starts building up, the system can also absorb an extra photon. This absorptive transition, from $N_{2}$ to $N_{4}$ leads to an intensitydependent increase in the absorption. Since these levels have a considerable energy spread due to the particle size distribution, the secondary absorptive transition occurs at the excitation wavelength $(390 \mathrm{~nm})$ as well as at PL wavelengths and is also responsible for the broad band PL quenching observed at excitation intensities of $\geq 1 \mathrm{~kW} / \mathrm{cm}^{2}$. Since this can only take place when the system is in an excited state, the nonlinearity observed in our samples is due to ESA. As mentioned earlier, the population in $N_{2}$ also decays to $N_{1}$ by a very slow nonradiative transition occurring over $\sim 2 \mathrm{~ms}$. This non-radiative transition is recorded by the non-degenerate pump-probe experiment and the carrier generation and relaxation time constants correspond to the decay constant of this transition. The reverse transition from $N_{4}$ to $N_{2}$ is fast and nonradiative. Since it occurs at a much faster rate than $\gamma_{\mathrm{c}}$, we ignore it while writing the rate equations. Denoting the carrier population in level $N_{i}$ by $n_{i}$ and total charge carriers by $N$, we can write the rate equations as

$$
\begin{aligned}
& \frac{\mathrm{d} n_{3}}{\mathrm{~d} t}=\beta I(t)-\Gamma n_{3}, \\
& \frac{\mathrm{d} n_{2}}{\mathrm{~d} t}=\gamma_{\mathrm{b}} n_{3}-\gamma_{\mathrm{c}} n_{2}, \\
& \frac{\mathrm{d} n_{1}}{\mathrm{~d} t}=-\beta I(t)+\gamma_{\mathrm{a}} n_{3}+\gamma_{\mathrm{c}} n_{2},
\end{aligned}
$$




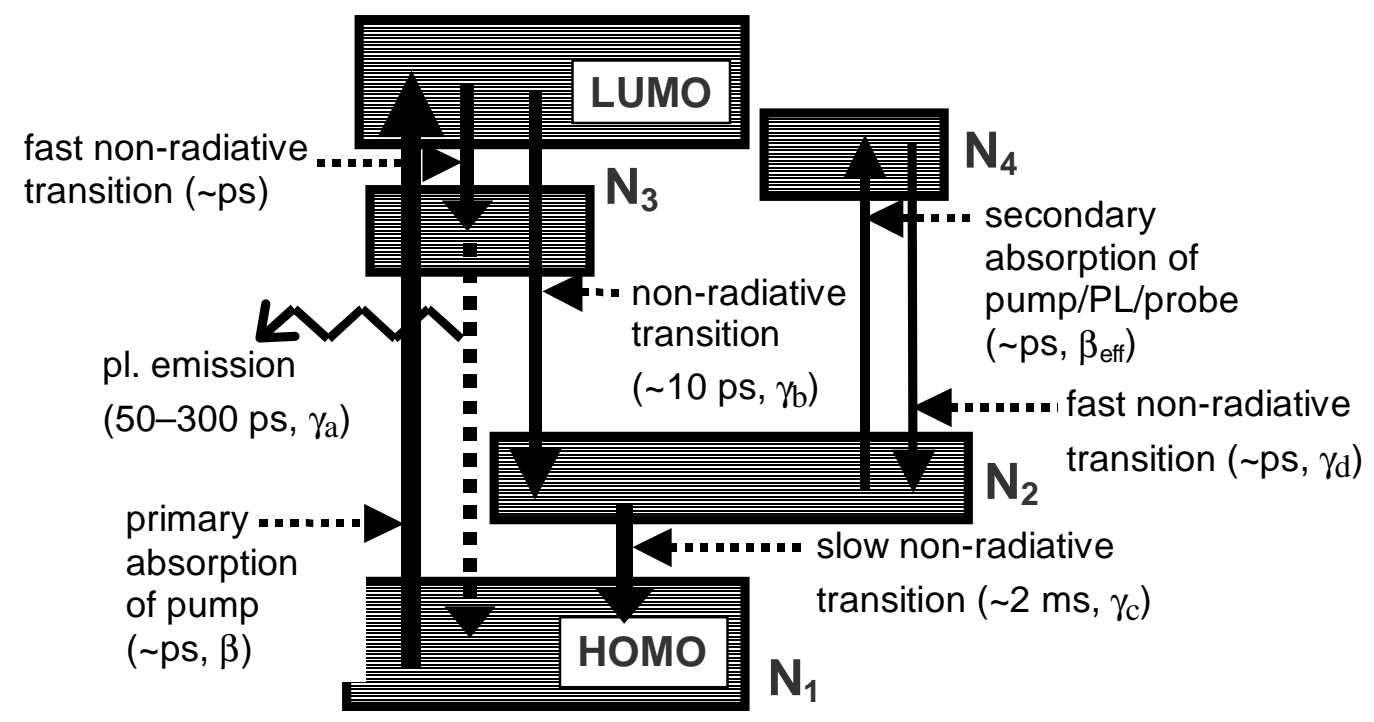

Figure 7. Schematic energy level diagram for the proposed 4-level model. States other than the HOMO and LUMO levels are generated by defects. The positions of the states indicated in the diagram do not denote the actual energies. Approximate time scales and decay rates (or absorption cross sections) for each transition are also shown.

$$
n_{1}+n_{2}+n_{3}=N
$$

where $\beta=\sigma n_{1} / \hbar \omega, \Gamma=\gamma_{\mathrm{a}}+\gamma_{\mathrm{b}}$, and $\sigma$ is the absorption cross-section for the optical transition from $N_{1}$ to $N_{3} . I(t)$ is the time dependent intensity of the pump beam with a square wave profile and time period, $T_{\mathrm{p}}(\approx 8 \mathrm{~ms})$ and amplitude, $I$. Here $I(t)=I$ for $t=0$ to $t=T_{\mathrm{p}}$ and $I(t)=I$ for $t=T_{\mathrm{p}}$ to $t=2 T_{\mathrm{p}}$. Assuming all the population is in the ground state initially $(t=0)$, we have

$$
n_{3}(t)=\frac{\beta I}{\Gamma}[1-\exp (-\Gamma t)]
$$

Substituting in (2) and solving for $t=0$

$$
\begin{aligned}
n_{2}(t) & =\frac{\beta I \gamma_{\mathrm{b}}}{\gamma_{\mathrm{c}} \Gamma}\left[1-\exp \left(-\gamma_{\mathrm{c}} t\right)\right] \\
& +\frac{\beta I \gamma_{\mathrm{b}}}{\Gamma\left(\gamma_{\mathrm{c}}-\Gamma\right)}\left[\exp (-\Gamma t)-\exp \left(-\gamma_{\mathrm{c}} t\right)\right]
\end{aligned}
$$

Since $\gamma_{c} \ll \Gamma,\left(\gamma_{c}-\Gamma\right) \cong-\Gamma$ and as the second term is very small compared to the first

$$
n_{2}(t)=\frac{\beta I \gamma_{\mathrm{b}}}{\gamma_{\mathrm{e}} \Gamma}\left[1-\exp \left(-\gamma_{\mathrm{c}} t\right)\right],
$$

during ON half cycle and

$$
n_{2}(t)=\frac{\beta I \gamma_{\mathrm{b}}}{\gamma_{\mathrm{c}} \Gamma}\left[\exp \left(\gamma_{\mathrm{c}} T_{\mathrm{p}}\right)-1\right] \exp \left(-\gamma_{\mathrm{c}} t\right),
$$

during OFF half cycle. In a pump-probe experiment for a weak probe beam, the variation in intensity as a function of distance within the sample (of thickness, $L$ ) is given by

$$
\frac{\mathrm{d} I_{2}}{\mathrm{~d} z}=-\sigma_{2} n_{2}(t) I_{2}
$$

where $\sigma_{2}$ is the cross-section for secondary absorption and $I_{2}$ the intensity of the top hat $\mathrm{CW}$ probe beam. Then the transmission of the probe beam is

$$
I_{\mathrm{tr}}=I_{2} \exp \left(-\sigma_{2} n_{2} L\right)
$$

provided the absorption coefficient for the probe beam is much smaller than that of the pump beam. Using (7), (8) and (10) we get

$$
I_{\mathrm{tr}} \propto 1-\frac{\beta I \gamma_{\mathrm{b}}}{\gamma_{\mathrm{c}} \Gamma}\left[1-\exp \left(-\gamma_{\mathrm{c}} t\right)\right],
$$

during $\mathrm{ON}$ half period and

$$
I_{\mathrm{tr}} \propto 1-\frac{\beta I \gamma_{\mathrm{b}}}{\gamma_{\mathrm{c}} \Gamma}\left[\exp \left(\gamma_{\mathrm{c}} T_{\mathrm{p}}\right)-1\right] \exp \left(-\gamma_{\mathrm{c}} t\right)
$$

during OFF half period. Equations (11) and (12) can be written in the form

$$
y=y_{0}+A \exp (-B t),
$$

where $y_{0}$ and $A$ are constants that are different for the $\mathrm{ON}$ and OFF half cycles, whereas the decay constant, $B\left(\equiv \gamma_{\mathrm{c}}\right)$, is the same in both cases. Figure 2 shows the experimental pump-probe data (squares) and the fit (solid line) based on our model for both ON and OFF half cycles. In view of the excellent agreement between the experimental data and the fitted curve, it is clear that our model explains all the observed optical features in the $\mathrm{CdS}$ : $\mathrm{ZnO}$ nanocomposite as well as the nano-CdS films. This is the first 
(albeit semi-quantitative) model for ESA in II-VI semiconductor thin films occurring at low fluences. It was not possible to study other non-radiative transitions using our set up as the fastest decay constant that was possible to measure was $\sim 100 \mu \mathrm{s}$. The fact that the PL yield in the nanocomposite system peaks at a specific $\mathrm{CdS}: \mathrm{ZnO}$ ratio is explained by a stronger coupling between two pairs of levels and faster decay rates for that particular composition. $\mathrm{ZnO}$ appears to play a crucial role in optimizing the optical properties by controlling the interaction between CdS nanoparticles.

In brief, optical properties such as photoluminescence quantum yield and nonlinear optical coefficients can be improved significantly through the formation of a twocomponent nanocrystalline semiconductor system with an optimal composition. One photon resonant nonlinear optical process discussed here can adversely affect their optoelectronic response. The high effective nonlinear coefficient and fast recovery observed in the nanocomposite, $\mathrm{CdS}: \mathrm{ZnO}$ thin films, make them important for applications related to optical limiting of $\mathrm{CW}$ and high rep-rate pulsed lasers. Due to their flat NLO response, these films can also be used as broad-band optical limiters for UV- visible and near infrared wavelengths in pump-probe geometry. One-photon resonant nonlinear optical response could be explained using a semiquantitative 4-level model.

\section{Acknowledgements}

Work presented here was done in collaboration with Parinda Vasa and Prof. P Ayyub, Department of CMP\&MS, Tata Institute of Fundamental Research, Mumbai.

\section{References}

Ayyub P, Chandra R, Taneja P, Sharma A K and Pinto R 2001 Appl. Phys. A73 67

Ayyub P, Vasa P, Taneja P, Banerjee R and Singh B P $2005 \mathrm{~J}$. Appl. Phys. 97104310

Taneja P, Vasa P and Ayyub P 2002 Mater. Lett. 54343

Vasa P, Taneja P, Ayyub P, Singh B P and Banerjee R 2002 J. Phys. Cond. Matter 14281

Vasa P, Ayyub P and Singh B P 2005 Appl. Phys. Lett. 87 063104

Woggon U 1997 Optical properties of semiconductor quantum dots (Berlin: Springer) 